\title{
HIGH-FIDELITY SIMULATIONS OF A HIGH-PRESSURE TURBINE VANE SUBJECT TO LARGE DISTURBANCES: EFFECT OF EXIT MACH NUMBER ON LOSSES
}

\author{
Yaomin Zhao* \\ Department of Mechanical Engineering \\ University of Melbourne \\ Melbourne, VIC 3010, Australia \\ Email: yaomin.zhao@unimelb.edu.au
}

\author{
Richard D. Sandberg \\ Department of Mechanical Engineering \\ University of Melbourne \\ Melbourne, VIC 3010, Australia \\ Email: richard.sandberg@unimelb.edu.au
}

\begin{abstract}
We report on a series of highly resolved large-eddy simulations of the LS89 high-pressure turbine (HPT) vane, varying the exit Mach number between $M a=0.7$ and 1.1. In order to accurately resolve the blade boundary layers and enforce pitchwise periodicity, we for the first time use an overset mesh method, which consists of an O-type grid around the blade overlapping with a background H-type grid. The simulations were conducted either with a synthetic inlet turbulence condition or including upstream bars. A quantitative comparison shows that the computationally more efficient synthetic method is able to reproduce the turbulence characterictics of the upstream bars. We further perform a detailed analysis of the flow fields, showing that the varying exit Mach number significantly changes the turbine efficiency by affecting the suction-side transition, blade boundary layer profiles, and wake mixing. In particular, the $M a=1.1$ case includes a strong shock that interacts with the trailing edge, causing an increased complexity of the flow field. We use our recently developed entropy loss analysis (Zhao and Sandberg, GT201990126) to decompose the overall loss into different source terms and identify the regions that dominate the loss generation. Comparing the different Ma cases, we conclude that the main mechanism for the extra loss generation in the $M a=1.1$ case is the shock-related strong pressure gradient interacting with the turbulent boundary layer and the wake, resulting in significant turbulence production and extensive viscous dissipation.
\end{abstract}

\footnotetext{
*Address all correspondence to this author.
}

\section{NOMENCLATURE \\ Symbols}

$C_{a x}$ Axial chord length.

$L_{S} \quad$ Turbulence integral length scale.

$M a$ Mach number.

$M a_{i s} \quad$ Isentropic Mach number.

$P_{t}^{i} \quad$ Inlet total pressure.

$P^{o} \quad$ Outlet pressure.

$P_{w} \quad$ Blade surface pressure.

$\mathrm{Pr}$ Prandtl number.

$R$ Gas constant.

$R e$ Reynolds number.

$T_{u} \quad$ Turbulence intensity.

$T_{t}^{i} \quad$ Inlet total temperature.

$T_{w} \quad$ Blade surface wall temperature.

$U^{i}$ Inlet mean velocity.

$x$ Axial coordinate, normalized by axial chord.

$y$ Pitchwise coordinate, normalized by axial chord.

\section{Acronyms}

CFD Computational fluid dynamics

DNS Direct numerical simulation

HPT How-pressure turbine

LES Large-eddy simulation

RANS Reynolds averaged NavierStokes

SGS Sub-grid scale 


\section{INTRODUCTION}

The high-pressure turbine (HPT) vane, immediately downstream of the combustion chamber, typically experiences high levels of unsteadiness and turbulence due to the combustor, and the highest temperatures, pressures, and velocities anywhere in the engine. Considering the extreme operating conditions, a detailed understanding of the aerothermal behavior of the HPT can be extraordinarily challenging. To date, the majority of research on HPT performance is carried out by physical tests (e.g. [1,2]), that are costly. Moreover, physical testing can seldom dissect the range of physical phenomena impacting the performance of a design. Alternatively, the well-known Reynolds Averaged NavierStokes (RANS) is a key design tool due to its low-computational cost. However, RANS (or unsteady RANS) usually suffers from accuracy issues due to challenges in modeling turbulence, which limits its true benefit especially in the presence of complex mixing processes [3].

High fidelity simulations, including Direct Numerical Simulation (DNS) and Large-Eddy Simulation (LES), have been shown to be very accurate in simulating turbomachinery flows [4]. By resolving most of the energy-containing turbulent scales in the unsteady flow field in an HPT, LES are able to provide insight into complex flow physics including the impact of curvature and pressure gradient on boundary layers, and the two-way interaction between incoming turbulence and boundary layer transition $[5,6]$. While the various previous high fidelity simulations on HPT mainly focused on the effects of inlet turbulence [5,7-10], other factors including the influence of exit Mach number are also of great interest. In particular, the HPT vane typically works at transonic Mach numbers which lead to shock waves that interact with boundary layers, and changing the pressure ratio between the outlet and inlet can significantly affect the aerothermal behavior by altering the location and strength of the shocks.

In the present study, we perform highly resolved LES of an HPT vane working at different exit Mach numbers, and the outline of the present study is summarized as follows. In order to handle the complex flow physics caused by the strong shock in the relatively high Mach number case, new features, such as an overset method and a shock filtering, have been introduced in the HPT setup. Thereafter, the highly resolved flow fields have been obatained and validated, and the Mach number effects have been identified based on the comparison between the cases. Furthermore, the recently proposed entropy loss analysis method [11] has been applied to the HPT data to understand the physical mechanisms responsible for the different aerothermal behaviors.

\section{NUMERICAL SETUP}

We apply the in-house structured compressible NavierStokes solver, HiPSTAR, to perform highly resolved LES of the VKI LS89 HPT vane [1]. HiPSTAR was developed and optimized for turbomachinery studies on the latest massively paral- lel high-performance computing systems, and the details of the code can be found in Sandberg et al. [12]. It is noted here that one key feature of HiPSTAR is that the numerical methods used ensure high-order accuracy of both spatial and temporal discretizations. In the present simulations, a fourth-order wavenumberoptimized compact finite difference scheme [13] is applied for spatial discretization, and a ultra-low storage frequency optimized fourth-order explicit Runge-Kutta method [14] is used for time integration. HiPSTAR has been applied in DNS and LES of various components, including turbines and compressors [12,15-18]. In particular, a series of LES of the VKI HPT vane [1] was performed at $R e=5.7 \times 10^{5}$ and $M a=0.9$ [5], covering a wide range of inlet turbulent states including turbulence intensities of $T_{u}=6.5 \% \sim 20 \% U^{i}$ and integral length scales of $L_{S}=5 \% \sim 20 \% C_{a x}$, where

$$
T_{u}=\sqrt{\frac{u^{\prime 2}+v^{\prime 2}+w^{\prime 2}}{3}} .
$$

While the previous simulations [5] mainly consider the effects of inlet turbulence, the present study focuses on the effects of varying exit Mach numbers on the aerothermodynamics of the HPT vane. In particular, the high Mach number case introduced in the present study is expected to include a strong shock and will show obvious trailing-edge shock interactions, which will cause additional complexity of the flow field and change the heat transfer and kinetic loss of the turbine. In order to deal with the complex physics, the shocking-filtering method proposed by Bogey et al. [19] is applied to resolve the strong shock in the high Mach number case. In addition, an overset method has been introduced to the HPT simulations for a simplified and more efficient grid generation, and the case setup and the validity of synthetically generated inlet turbulence are extensively scrutinized and discussed in the following sections.

\section{Overset Mesh Method}

In previous simulations on turbomachinery components with HiPSTAR, the structured meshes in the computational domain were decomposed into different blocks as shown in Fig. 1(a). This multi-block configuration included an O-type grid around the turbine blade and an H-type grid away from the blade, and the characteristic interface conditions (CIC) [20] were applied to permit non-orthogonality of the computational grid at block interfaces, without loss of accuracy. It is noted that this multi-block configuration has been proven successful in previous simulations, shedding light on detailed physics by generating highly resolved flow fields. However, for numerical simulations of the present cases, especially with the existence of the strong shock in the high Mach number case, the CIC can suffer from severe instability issues when the shock interacts with the block interfaces, shown as dashed lines in Fig. 1(a). 

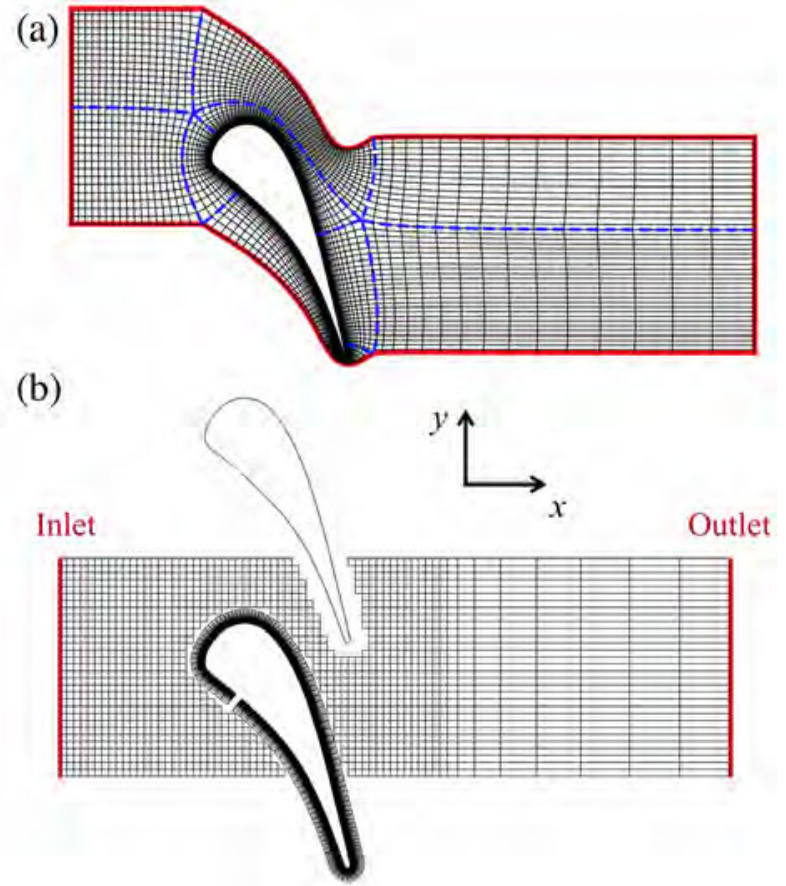

FIGURE 1. CONFIGURATION OF THE LS89 HPT VANE CASE. THE MESH IS SHOWN EVERY 15 POINTS FOR CLARITY. (a) THE 9-BLOCK SETUP, WITH THE BLUE DASHED LINES INDICATING BLOCK INTERFACES; (b) THE OVERSET SETUP.

To overcome this difficulty, the overset method, which originates from Chesshire and William [21], is applied in the present HPT simulations. As shown in Fig. 1(b), the newly introduced overset configuration includes a background H-type grid, which allows for pitchwise periodic boundary conditions, and an $\mathrm{O}$ type grid around the blade, enabling adequate resolution around the blade boundary layer especially at leading and trailing edges. The H-type and O-type grids overlap with each other, and at the overlapping boundaries, continuity conditions are imposed as variables are interpolated with a fourth-order Lagrangian method and communicated between the grids. The principal advantage of this overset configuration is that the mesh generation process for the complex geometry of an HPT is straightforward, as the orthogonality of the computational grid is easy to enforce. Furthermore, this overset configuration avoids the numerical inaccuracy and the instability issues caused by the CIC at the multi-block interfaces as in Fig. 1(a), especially with the stong shocks in the high Mach number case in the present study.

\section{Case Configuration}

With the newly introduced overset configuration as shown in Fig. 1(b), HPT cases with three different Mach numbers, varying from $M a=0.7$ to $M a=1.1$, are considered in the present study.
TABLE 1. PARAMETERS OF DIFFERENT CASES.

\begin{tabular}{ccccccl}
\hline Cases & $M a$ & $\begin{array}{c}R e_{2} \\
\left(\times 10^{5}\right)\end{array}$ & $\begin{array}{c}\text { Inlet } \\
\text { Turb. }\end{array}$ & $T_{u} / U^{i}$ & $L_{S} / C_{a x}$ & Grids points \\
\hline A & 0.7 & 5.2 & Syn. & $20 \%$ & $10 \%$ & $6.3 \times 10^{8}$ \\
B & 0.9 & 5.7 & Syn. & $20 \%$ & $10 \%$ & $6.3 \times 10^{8}$ \\
C & 1.1 & 5.8 & Syn. & $20 \%$ & $10 \%$ & $6.3 \times 10^{8}$ \\
\hline D & 0.9 & 5.7 & Syn. & $6.5 \%$ & $5 \%$ & $3.8 \times 10^{8}$ \\
E & 0.9 & 5.7 & Bar & $7 \%$ & $8 \%$ & $14.5 \times 10^{8}$ \\
\hline
\end{tabular}

As shown in Tab. 1, five cases are divided into two groups. The first group, the cases $\mathrm{A}-\mathrm{C}$, introduce high-level inlet turbulence ( $20 \%$ of the inlet mean velocity $U^{i}$ ) with large integral length scale ( $10 \%$ of the blade axial chord length $C_{a x}$ ), which is comparable to realistic fluctuations at combustor exits. With the same turbulent fluctuations at the inlet, the cases $\mathrm{A}-\mathrm{C}$ focus on the effects of the exit Mach number on the aerothermodynamics of the HPT vane.

In addition, in order to validate the current setup including the mesh quality and the accuracy of the numerical methods, cases D and E apply inlet turbulence at levels similar to the MUR224 case from the VKI experiment [1]. Specifically, the case $\mathrm{D}$ employs a widely used synthetic turbulence generation method [22], while the incoming turbulence in case $\mathrm{E}$ is generated by introducing cylindrical bars upstream the HPT vane, as shown in Fig. 2. It is noted that the setup of case $\mathrm{E}$ is to resemble the experiment, and the simulation is much more challenging compared to the other cases with sythetic turbulence, as a much larger computational domain is required in both the streamwise and spanwise directions to resolve the development of the cylindrical wakes. As shown in Tab. 1, the number of the total grid points used in case $\mathrm{E}$ is $14.5 \times 10^{8}$, which is too expensive to allow for a parameter sweep as in cases A - C.

For all of the five cases in Tab. 1, the fully compressible Navier-Stokes equations are solved with HiPSTAR, and the subgrid scale (SGS) contribution is provided by the standard WALE model [23]. A no-slip isothermal wall boundary condition is specified at the blade surface, with the wall temperature set as $300 \mathrm{~K}$ which is same as in the VKI experiments, and periodic boundary conditions are applied in the pitchwise and spanwise directions. Furthermore, the non-reflective zonal characteristic boundary condition [24] is used at the outlet, and the Riemann invariant boundary conditions have been used at the inlet. The Mach number is varied between cases by changing the ratio of the outlet static pressure $P^{o}$ and the inlet total pressure $P_{t}^{i}$. To be specific, the inlet conditions remain constant and the exit static 


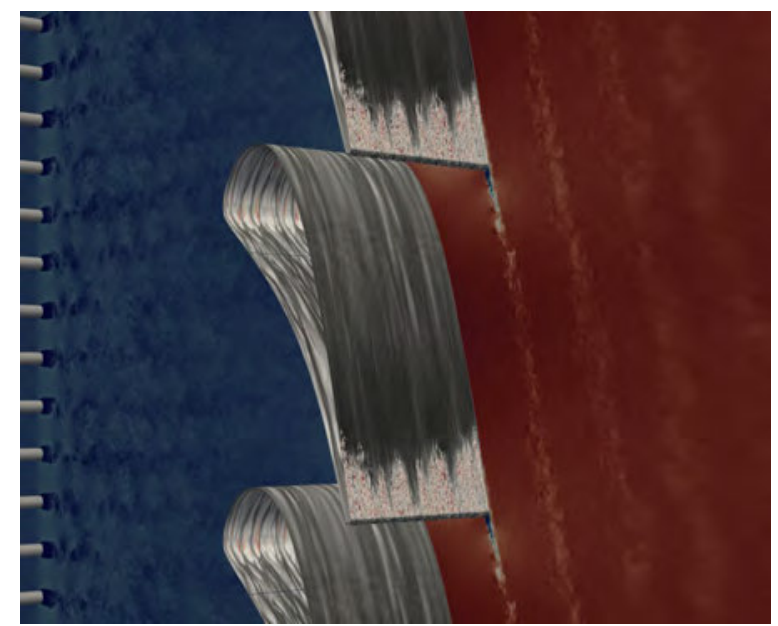

FIGURE 2. CASE E WITH BARS TO GENERATE INCOMING TURBULENCE. THE CONTOUR OF VELOCITY MAGNITUDE IS SHOWN ON THE STREAMWISE-PITCHWISE PLANE-CUT, WHILE THE TEMPERATURE IS PRESENTED AROUND THE BLADE.

pressure is changed in these cases. As a result, the Reynolds number at the vane exit $R e_{2}$ slightly deviates between the cases as shown in Tab. 1, while the inlet Reynolds number stays constant. However, as the Reynolds number differences are relatively small, especially between the cases B and C which focus on the transonic behavior around the blade, we remark that the Reynolds number effects in the present study is not significant and the Mach number effect is the dominating factor.

Moreover, the cases share the same grid resolution in both the background H-type and the O-type grids. In particular, the grid resolution around the blade boundary layer for case B is shown in figure 3 . The grid spacings on the blade surface in the tangential, wall-normal, and spanwise directions are normalized with the local viscous length scale $\delta_{v}=v / u_{\tau}$ and denoted as $\Delta s^{+}, \Delta n^{+}$, and $\Delta z^{+}$, respectively. Here $v$ is the molecular viscosity, and $u_{\tau}=\sqrt{\tau_{w} / \rho}$ is the wall friction velocity with the wall shear stress $\tau_{w}$ and density $\rho$. Although the grid sizes vary along the blade surface, they remain at a relatively low level, and the grid spacings averaged around the blade surface are given as $\left\langle\Delta s^{+}\right\rangle \approx 28.6,\left\langle\Delta n_{w}^{+}\right\rangle \approx 1.97$, and $\left\langle\Delta z^{+}\right\rangle \approx 10.9$. The extraordinarily fine resolution in the spanwise and streamwise directions are believed to be critical to accurately predict the development of the blade boundary layer under the large-scale incoming turbulence [4]. Moreover, as the Reynolds number deviations in the cases are not significant as shown in Tab 1, the grid resolutions in the present cases with different Mach numbers are at similar levels.

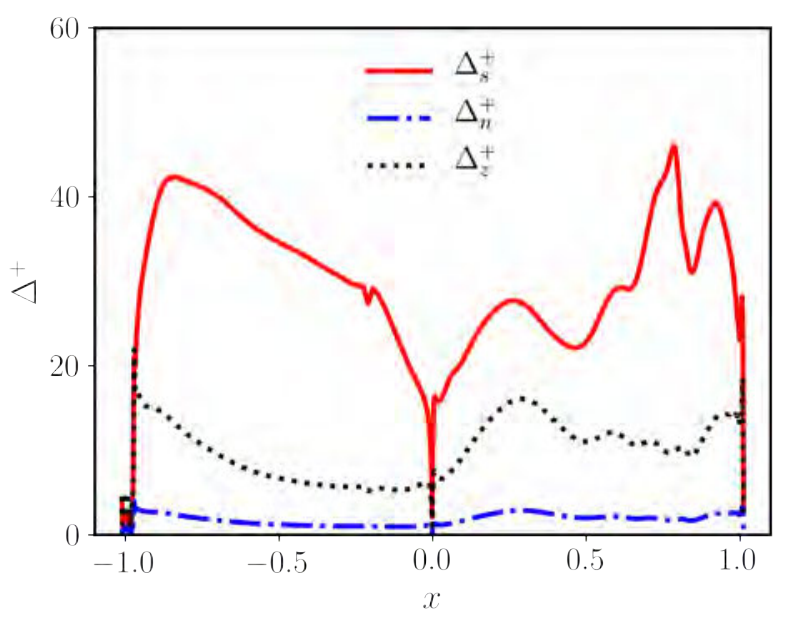

FIGURE 3. GRID RESOLUTION AROUND THE BLADE BOUNDARY LAYER NORMALIZED IN WALL UNITS IN CASE B. PRESSURE SIDE: $x<0$; SUCTION SIDE: $x>0$.

\section{VALIDATION OF INLET TURBULENCE}

Generation of realistic inlet turbulence is critical for scaleresolving computations of spatially developing turbulent flows, and different methods have been proposed in the past few decades [25]. One of the most popular methods, the synthetic digital filtering method [22], is compared in the present study against the more 'realistic' turbulence generated by upstream bars. Based on the comparison between cases D and E, the objective here is to rigorously study the effects of the chosen turbulence generation methods on the HPT performance.

First of all, the convective heat transfer coefficient

$$
H=-\frac{\mu c_{p}}{\operatorname{Pr}\left(T_{t}^{i}-T_{w}\right)} \frac{\partial T}{\partial n}
$$

from cases $\mathrm{D}$ and $\mathrm{E}$ is compared with experimental data of the MUR224 case [1]. Here, $\operatorname{Pr}$ is the Prandtl number, $c_{p}$ is the specific heat, and $T_{t}^{i}$ and $T_{w}$ are inlet total temperature and wall temperature, respectively. As shown in Fig. 4, the numerical simulations are able to accurately predict the wall heat transfer coefficient for most of the blade boundary layer. Only slight deviations are observed on the suction-side blade, downstream of the boundary layer transition. This is because the wall heat-flux level in the transitonal boundary layer is affected by the freestream turbulence intensity, and it is quite challenging to reproduce the exact turbulence states in the numerical simulations as the turbulence length scales or dissipation rate were not provided in the experiments [7]. However, the close agreement between the numerical results and the experimental data in the transitional boundary layer suggests the simulations are able to reproduce 


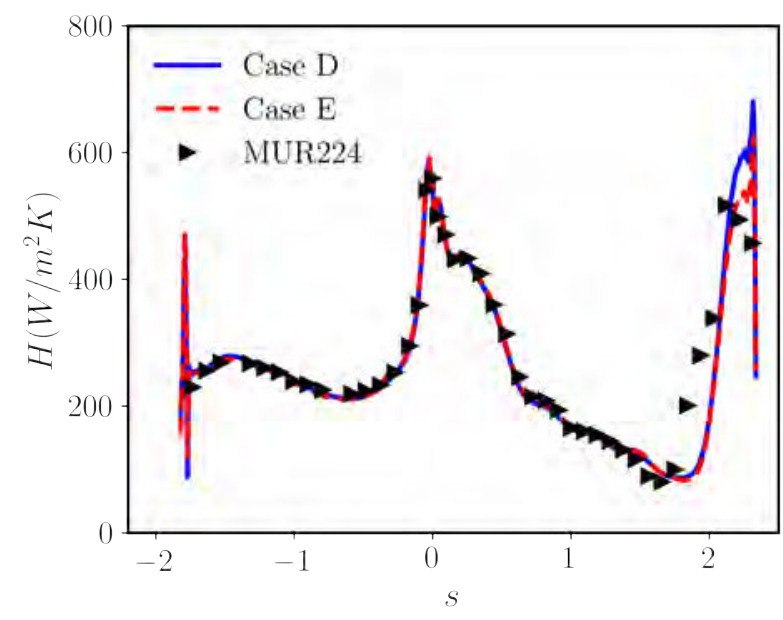

FIGURE 4. WALL HEAT TRANSFER COEFFICIENT AROUND THE BLADE BOUNDARY LAYER. PRESSURE SIDE: $s<0$; SUCTION SIDE: $s>0$.

the experimental setup.

The high-order statistics of the turbulence in cases D and E are further investigated to study the effects of different turbulence generation methods. In Fig. 5, the turbulence spectra at different locations, both upstream the HPT leading edge and in the wake region downstream of the trailing edge, are presented. Compared to the turbulence from the bar wakes, the synthetic turbulence generated in case D follows the slope of the inertial range for an extraordinary long range upstream of the HPT vane, as shown in Fig. 5(a). This indicates that the turbulence by the digital filter method introduces an artificial inertial scaling which is not possible in realistic turbulence. However, this effect only affects the small scales, which do not significantly influence the mean flow behavior through the HPT vane, as shown in Fig. 4. Furthermore, as the turbulence develops downstream the vane, the spectra from cases D and E become much more similar and in the wake are in close agreement, including both the inertial scaling and the vortex shedding frequency indicated by the lowfrequency peak shown in Fig. 5(b).

As a summary, the turbulence generated by the synthetic method initially introduces an articial scaling at small scales, but the turbulence energy cascade develops further downstream and the mean flow statistics seem not to be affected by the choice of turbulence generation methods. Therefore, considering the significantly lower computational cost of case D as suggested in Tab. 1, the synthetic method has been applied to study the Mach number effects.
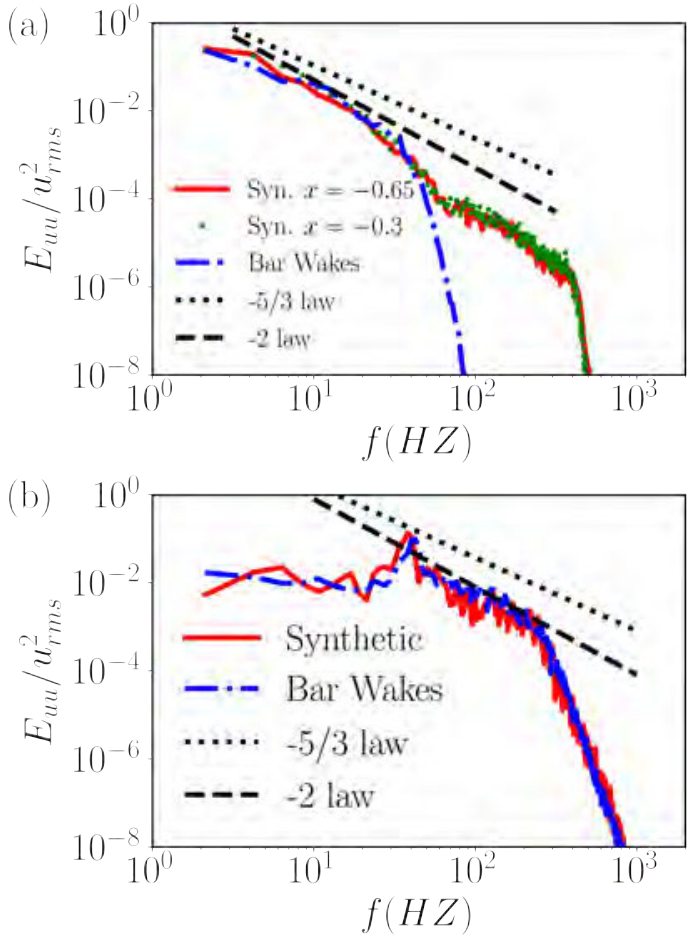

FIGURE 5. TURBULENCE SPECTRA GENERATED BY DIFFERENT METHODS. (a) UPSTREAM HPT VANE AT $x=-0.65$; (b) DOWNSTREAM HPT VANE IN THE WAKE AT $x=1.03$.

\section{RESULTS \\ Qualitative Overview}

An overview of the flow fields from cases $\mathrm{A}-\mathrm{C}$ is first given in Fig. 6, in which the contour of the mean pressure $P$ is shown on a spanwise plan-cut and the wall-normal fluctuating velocity $u_{n}^{\prime}$ is shown on a surface close to the blade. It is obvious that the mean pressure distribution through the HPT vane deviates significantly in cases with different Mach numbers. In particular, the difference of $P$ distribution is mainly downstream of the throat region, where a strong shock is present in Fig. 6(c) in the $M a=1.1$ case.

Furthermore, the $u_{n}^{\prime}$ contour on the surface parallel to the wall can indicate the laminar-turbulent transition on the suctionside boundary layer. The incoming turbulence interacts with the blade leading edge, leaving the boundary layer perturbed. Under the pressure gradient and the high-curvature effects of the blade surface, the fluctuations inside the boundary layer then develop while convecting downstream, and finally initiate transition to turbulence [6]. As shown in Fig. 6, the transition onsets vary between cases with different Mach numbers. Considering that the inlet turbulence and the numerical configuration are the same for all the cases, the $M a$ influence on the transition onsets is likely to be due to the pressure distribution in the HPT suction-side 


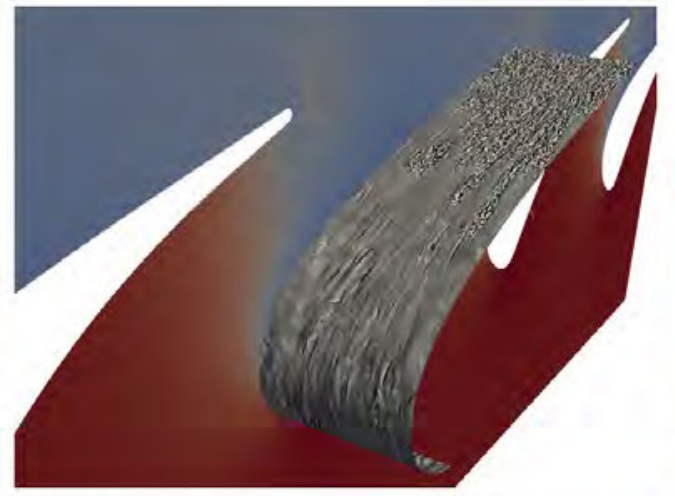

(a)

$P / P_{t}^{i}$

$T^{1.0}$

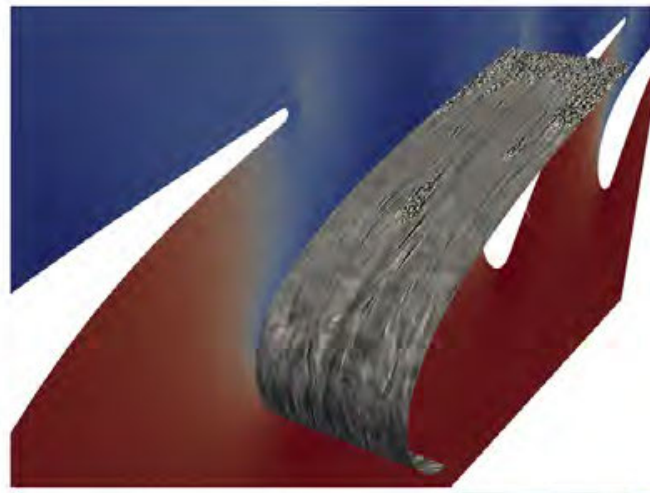

(b)

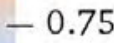

0.50
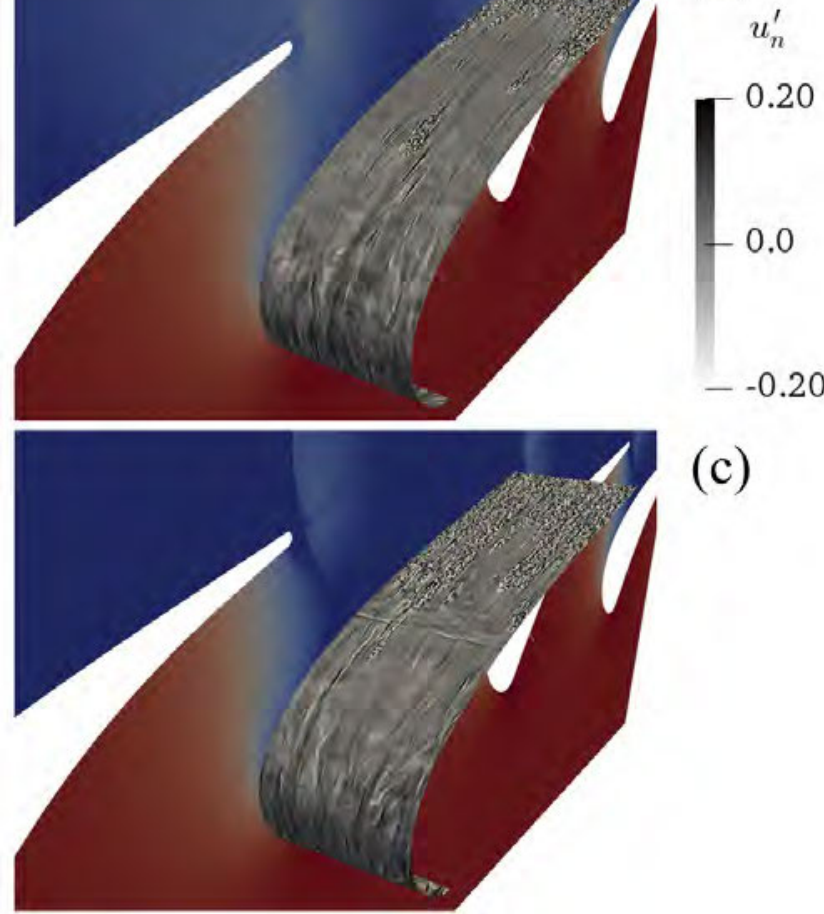

(c)

FIGURE 6. OVERVIEW OF THE FLOW FIELDS FROM (a) CASE A; (b) CASE B; AND (c) CASE C. THE MEAN PRESSURE $P$ IS SHOWN ON THE SPANWISE PLANE-CUT AND THE WALLNORMAL FLUCTUATING VELOCITY $u_{n}^{\prime}$ IS SHOWN ON A SURFACE CLOSE TO THE BLADE.

boundary layer, which will be quantitatively analyzed in the following sections.

\section{Quantification of Mach Number Effects}

The effects of the exit Mach number on the aerothermal behavior of the HPT have been investigated by comparing between the cases $\mathrm{A}-\mathrm{C}$. The isentropic Mach number $M a_{i s}$, which is
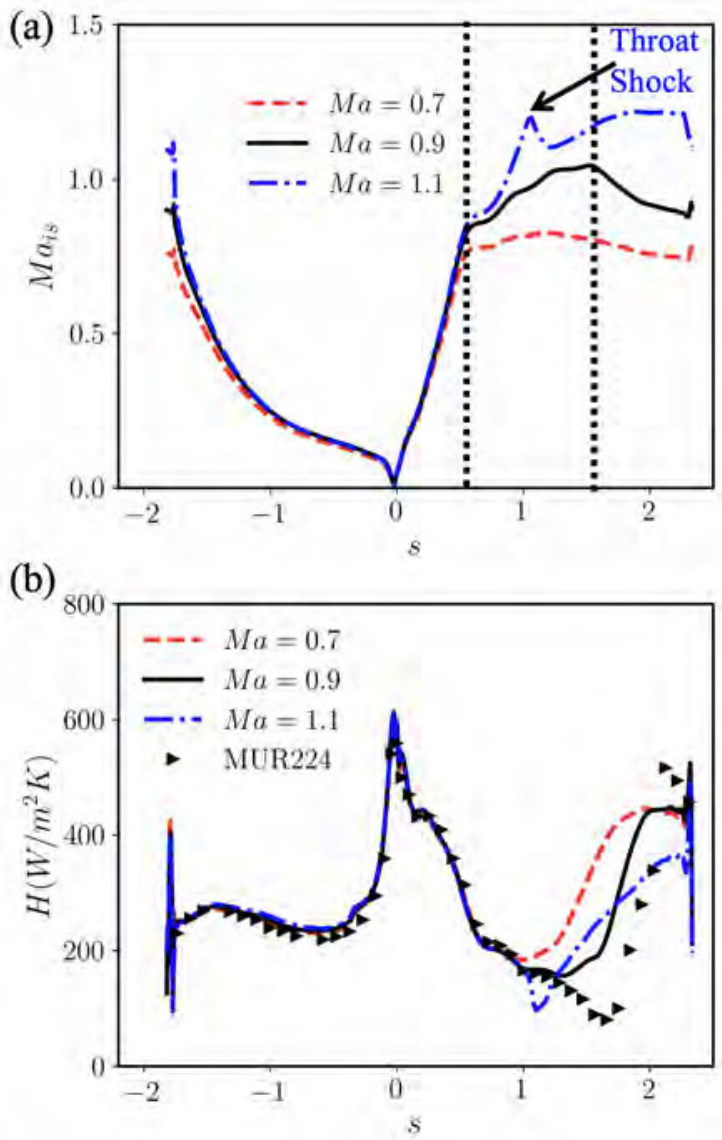

FIGURE 7. (a) ISENTROPIC MACH NUMBER AROUND THE BLADE BOUNDARY LAYER; (b) WALL HEAT TRANSFER COEFFICIENT AROUND THE BLADE BOUNDARY LAYER. PRESSURE SIDE: $s<0$; SUCTION SIDE: $s>0$.

related to the pressure distribution around the blade $P_{w}$ as

$$
M a_{i s}=\left(\frac{2}{\gamma-1}\left(\left(P_{w} / P_{t}^{i}\right)^{-\frac{\gamma-1}{\gamma}}-1\right)\right)^{\frac{1}{2}}
$$

is shown in Fig. 7(a), with $\gamma=1.4$ denoting the specific heat ratio. We divide the suction-side boundary layer into different stages by the vertical black dotted lines, and the different Mach number cases show similar pressure distributions on the pressure-side and the first stage of the suction-side boundary layers. This indicates that the pressure distribution upstream of the throat region of the HPT vane is hardly affected by the pressure ratio around the working conditions, and the corresponding boundary layers are dominated by a strong favourable pressure gradient (FPG).

Downstream of the throat, the suction-side boundary layers show significant differences for the different Mach number cases. 
To be specific, the pressure gradient in the $M a=0.7$ case is negligible as indicated by the plateau-like section of $M a_{i s}$. In case $B$ with $M a=0.9$, however, the suction-side boundary layer is subject to a favourable and then an adverse pressure gradient (APG), which are divided by the second vertical dotted line in Fig. 7(a). Furthermore, a strong shock appears in the $M a=1.1$ case, as indicated by the spike shown by the $M a_{i s}$ distribution. The differences of the pressure distribution in these cases not only affect the development of the suction-side boundary layer downstream the throat, but also cause distinctive wake-mixing behaviors near the blade trailing edge.

The behaviors of the blade boundary layers can be demonstrated by the wall heat transfer coefficient as shown in Fig. 7(b). In agreement with the $M a_{i s}$ distribution, the differences on the pressure-side and the first stage of the suction-side boundary layers are negligible among the cases, whereas the heat transfer coefficient distributions on the suction-side show significant deviations further downstream. Particularly, the transition onsets in cases with different Mach numbers are directly influenced by the pressure distribution as suggested by Fig. 7(a). It is noted that the increase of heat flux in the $M a=0.9$ case is later than in the $M a=0.7$ case, which is because the stabilizing effect of the FPG in the second stage of the suction-side boundary layer effectively delays the transition onset [6]. Furthermore, the transition onset in the $M a=1.1$ case is immediately downstream of the strong shock. Moreover, the earlier transition onset in case B compared to the experimental data at the same Mach number (and cases D and E in Fig. 4) is due to the higher level of turbulence intensity at the freestream as presented in Fig. 7(b).

Associated with the different states of the blade boundary layers, the wake development of the HPT is also significantly altered among the cases with different Mach numbers. The total pressure downstream of the blade trailing edge $P_{t}^{o}$ is normalized by the inlet total pressure $P_{t}^{i}$ and plotted along the pitchwise cut at $x=1.4$ in Fig. 8 . It is noted that the total pressure loss is stronger at the wake center when the exit Mach number increases, suggesting a stronger mixing process caused by strong pressure gradient near the blade trailing edge. Moreover, compared to other cases, the $M a=1.1$ case shows an extensively wider wake region, and the total pressure loss at the freestream is also non-negligible. This is because the HPT nozzle is choked, and the strong shock induces irreversible loss in the flow past the vane.

\section{Entropy Loss Analysis}

The entropy generation process is closely related to the loss of efficiency of turbomachinery components [26], and an entropy breakdown method has recently been proposed to quantitatively investigate the physical mechanisms responsible for loss [11]. As shown in Eqn. (1), the entropy generation process in the HPT vane can be decomposed into several terms representing different

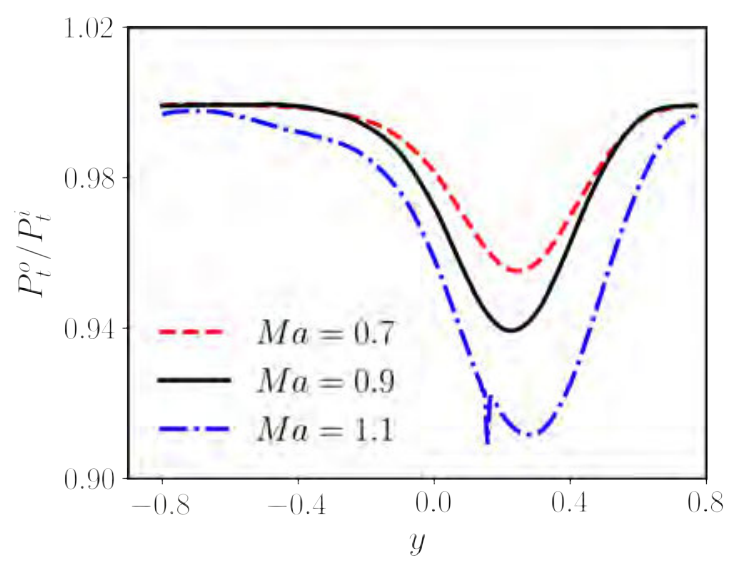

FIGURE 8. PITCHWISE TOTAL PRESSURE DISTRIBUTION $P_{t}^{o}$ AT $40 \% C_{a x}$ DOWNSTREAM OF THE BLADE TRAILING EDGE, NORMALIZED BY THE INLET TOTAL PRESSURE $P_{t}^{i}$.

physical mechanisms, including the mean flow viscous dissipation $V_{M}$, mean flow irreversible heat flux $H_{M}$, turbulence production $P_{R}$, turbulent heat flux $H_{R}$, advection of the turbulent kinetic energy $A_{k}$, SGS viscous dissipation $V_{S}$, and SGS heat flux term $H_{S}$

$$
\begin{aligned}
\widetilde{G}_{s}^{L E S}= & \underbrace{\frac{1}{\widetilde{T}} \sigma_{i j}(\widetilde{u}) \frac{\partial \widetilde{u}_{j}}{\partial x_{i}}}_{V_{M}}+\underbrace{\frac{c_{p} \mu}{P r} \frac{1}{\widetilde{T}^{2}}\left(\frac{\partial \widetilde{T}}{\partial x_{i}}\right)^{2}}_{H_{M}} \\
& +\underbrace{\frac{1}{\widetilde{T} \tau_{i j}^{R} \frac{\partial \widetilde{u}_{j}}{\partial x_{i}}}}_{P_{R}}-\underbrace{\frac{1}{\widetilde{T}} \frac{\partial}{\partial x_{i}}\left(q_{i}^{R}\right)}_{H_{R}}-\underbrace{\frac{1}{\widetilde{T}} \frac{\partial\left(\bar{\rho} \widetilde{u}_{i} k\right)}{\partial x_{i}}}_{A_{k}} \\
& +\underbrace{\frac{1}{\widetilde{T}} \widetilde{\tau_{i j}^{S S}} \frac{\partial \widetilde{u}_{j}}{\partial x_{i}}}_{V_{S}}-\underbrace{\frac{1}{\widetilde{T}} \frac{\partial}{\partial x_{i}}\left(q_{i}^{S G S}\right)}_{H_{S}} .
\end{aligned}
$$

Here, $\sigma_{i j}, \tau_{i j}^{R}, q_{i}^{R}, k, \tau_{i j}^{S G S}$ and $q_{i}^{S G S}$ denote viscous stresses, Reynolds stresses, turbulent heat flux, turbulent kinetic energy, SGS stresses and SGS heat flux, respectively. Moreover, ? represents a Favre-average operation, and the detailed derivation of Eqn. (1) can be found in Zhao and Sandberg [11]. These terms can be divided into three groups, including the mean flow effects $\left(V_{M}\right.$ and $\left.H_{M}\right)$, the entropy generation induced by turbulence fluctuations $\left(P_{R}, H_{R}\right.$ and $\left.A_{k}\right)$, and the SGS contribution by LES modelling $\left(V_{S}\right.$ and $\left.H_{S}\right)$. Furthermore, the volume integral of the total entropy generation $\widetilde{G}_{s}$ in the HPT vane can be linked to the total pressure loss [26] as

$$
F_{s}=-\dot{m} R \ln \left(P_{t, o} / P_{t, i}\right) \approx \int_{V} \widetilde{G}_{s} d V
$$


TABLE 2. QUANTIFICATION OF ENTROPY GENERATION TERMS IN CASES WITH VARYING MACH NUMBERS.

\begin{tabular}{lrrrr}
\hline $\begin{array}{c}\text { Terms } \\
\left(J K^{-1} s^{-1}\right)\end{array}$ & $M a=0.7$ & $M a=0.9$ & $M a=1.1$ & Shock \\
\hline$V_{M}$ & 0.1698 & 0.2181 & 0.2916 & 0.0017 \\
$H_{M}$ & 0.0809 & 0.0712 & 0.0656 & 0.0005 \\
$P_{R}$ & 0.2727 & 0.3710 & 0.6416 & 0.0552 \\
$H_{R}$ & -0.0084 & -0.0101 & 0.0205 & 0.0249 \\
$A_{k}$ & 0.0002 & -0.0073 & -0.0419 & -0.0141 \\
$V_{S}$ & 0.0138 & 0.0197 & 0.0337 & 0.0001 \\
$H_{S}$ & 0.0008 & 0.0001 & 0.00009 & 0.00002 \\
\hline$G_{S}$ & 0.529 & 0.663 & 1.011 & 0.0683 \\
$F_{S}$ & 0.512 & 0.665 & 1.065 & N.A. \\
\hline
\end{tabular}

where $\dot{m}$ is the mass flow rate through the vane and $R$ is the gas constant. Therefore, this entropy loss analysis method [11] can be applied to understand the aerothermodynamics of the cases with different Mach numbers.

This entropy analysis method has been applied to the cases A - C with varying exit Mach numbers. The LES data is averaged in time and in the spanwise direction, and the entropy generation terms in Eqn. (1) are integrated over the HPT vane for all three cases and the results are presented in Tab. 2. Similar to the previous results obatined for case D [11], several major observations can be drawn. First of all, the viscous effects are much stronger than the heat flux terms, as $V_{M}>H_{M}$ and $P_{R}>H_{R}$. Furthermore, the most important term is the turbulence production term $P_{R}$, which contributes more than $50 \%$ of the entropy generation for all cases. Finally, the SGS contribution is relatively small, $\left(V_{S}+H_{S}\right) / G_{S} \approx 3 \%$, indicating that the present LES flow is highly resolved.

To further understand the physical mechanisms, the spatial distributions of several of the major entropy generation terms in the HPT vane are investigated. The spatial distributions of $V_{M}$ in cases B and C are presented in Fig. 9(a) and (b), respectively, and a zoom-in view of the distribution in the $M a=1.1$ case is shown in Fig. 9(c). It is noted that the mean viscous dissipation is mainly in the blade boundary layers, which are dominated by strong shear effects, whereas for the rest of the flow domains we have $V_{M} \approx 0$. Furthermore, a comparison between the cases $\mathrm{B}$ and $\mathrm{C}$ shows that the shock in the $M a=1.1$ case also contributes to the viscous dissipation due to the strong velocity gradient in the affected region.

To quantitatively compare between the cases, the entropy
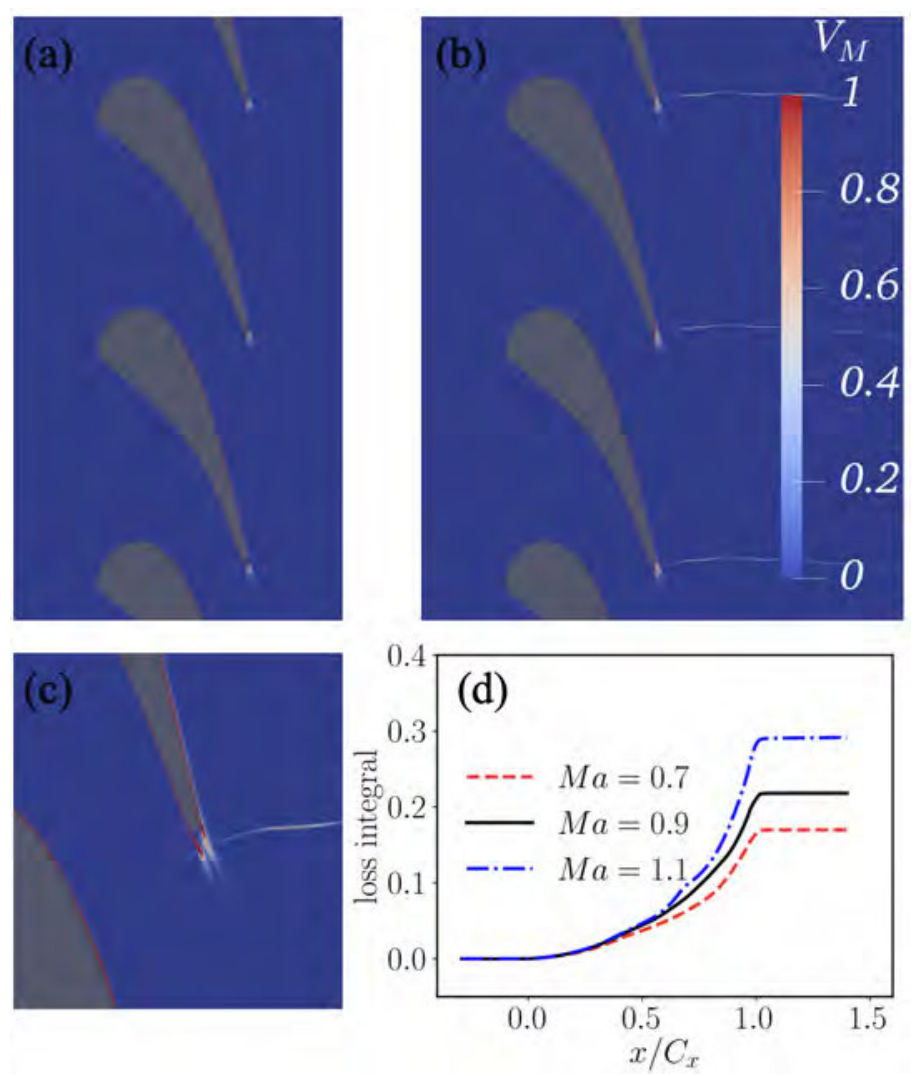

FIGURE 9. THE MEAN VISCOUS DISSIPATION TERM $V_{M}$. (a) SPATIAL DISTRIBUTION IN $M a=0.9$ CASE; (b) DISTRIBUTION IN $M a=1.1$ CASE; (c) ZOOM-IN VIEW OF THE $M a=1.1$ CASE; (d) STREAMWISE INTEGRAL FROM DIFFERENT CASES.

generation term $V_{M}$ can be integrated over the volume between the inlet plane to a downstream axial plane at $x$. As shown in Fig. 9(d), the increase of $V_{M}$ is distributed between the blade leading edge at $x=0.0$ and trailing edge at $x=1.0$, and the increase rates deviate between cases, especially downstream of the transition onsets indicated in Fig. 7(b). The reason for the higher increase rate in the $M a=1.1$ case is that the mean flow shear in the boundary layer is much stronger as shown in Fig. 10(a), which is related to the flow acceleration caused by the stronger pressure ratio. Moreover, it is also shown that the shock contribution downstream of the blade trailing edge is quite small compared to that in the blade boundary layer.

Similarly, the entropy generation induced by the turbulence production $P_{R}$ is also studied, and the corresponding results are shown in Fig. 11. For all of the cases, the $P_{R}$ term is concentrated in the turbulent boundary layer on the suction-side, which is downstream of the transition onset, and in the wake region, which is related to the strong vortex shedding downstream of the trailing edge. Meanwhile, the strong shock in the $M a=1.1$ case 


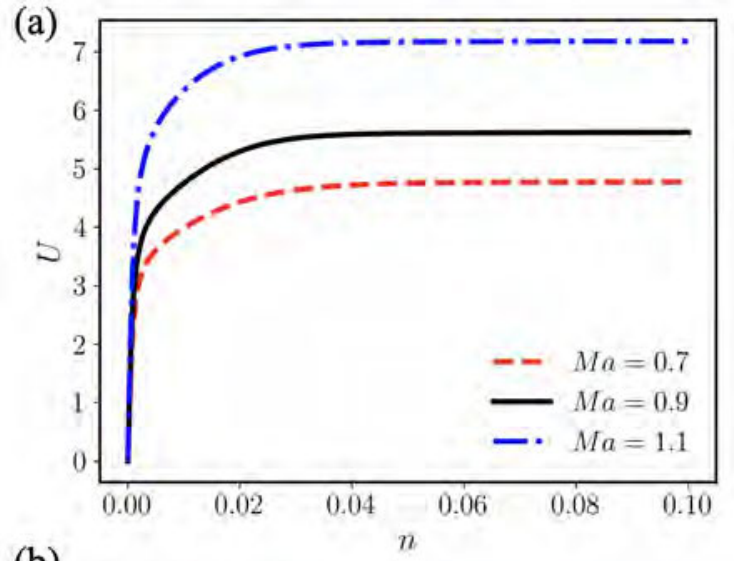

(b)

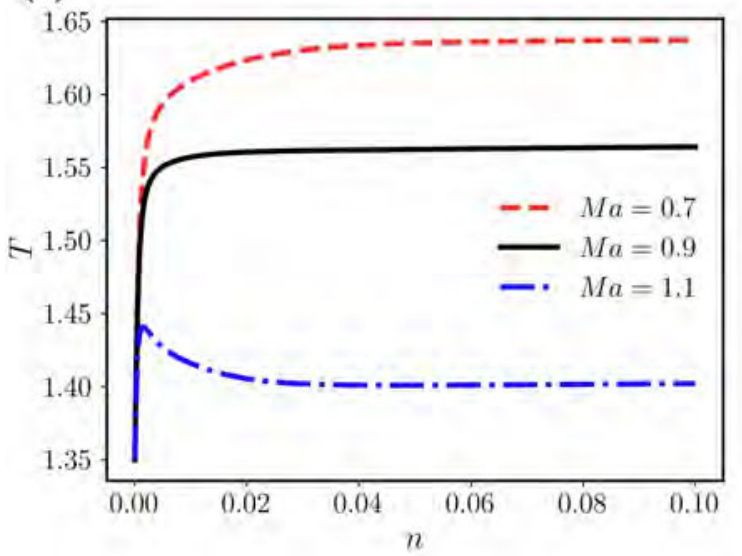

FIGURE 10. PROFILES PLOTTED AGAINST THE WALLNORMAL DISTANCE $n$ ON THE SUCTION-SIDE BOUNDARY LAYER NEAR THE TRAILING EDGE AT $x=0.97$. (a) WALLTANGENTIAL MEAN VELOCITY $U$; (a) MEAN TEMPERATURE $T$.

also has a non-negligible contribution as shown in Fig. 11(c). Furthermore, the volume integrals of the $P_{R}$ term in Fig. 11(d) provide a quantitative identification, showing that despite of the slight difference of the $P_{R}$ term in the turbulent boundary layer, most of the contribution is from the extensive wake mixing near the blade trailing edge which shows a strong Mach number dependency. This indicates that the turbulence generation caused by the vortex shedding is much stronger in the higher Mach number case.

Furthermore, the Mach number effects can be quantitatively analyzed by comparing between the entropy generation terms listed in Tab. 2. It is noted that the total entropy generation $G_{S}$, along with several major terms such as $V_{M}$ and $P_{R}$, is proportional to the exit Mach number. As discussed earlier, this is because the shear effects in the boundary layer and the wake mixing are stronger in cases at higher Mach numbers. However, the mean
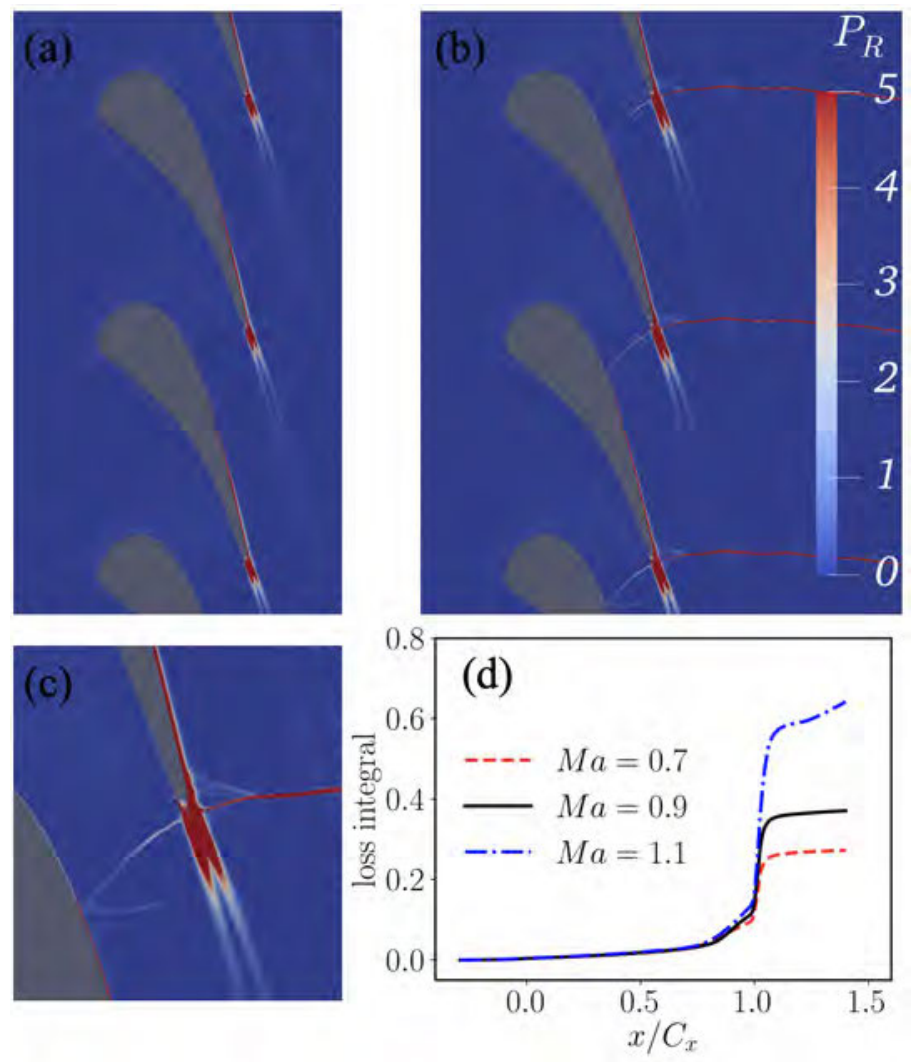

FIGURE 11. THE ENTROPY GENERATION TERM CAUSED BY TURBULENCE PRODUCTION $P_{R}$. (a) SPATIAL DISTRIBUTION IN $M a=0.9$ CASE; (b) DISTRIBUTION IN $M a=1.1$ CASE; (c) ZOOMIN VIEW OF THE $M a=1.1$ CASE; (d) STREAMWISE INTEGRAL FROM DIFFERENT CASES.

flow irreversible heat flux $H_{M}$ decreases while the $M a$ increase. This can be explained by the boundary layer profiles of temperature in Fig. 10(b), in which the temperature gradient in the $M a=1.1$ case is smaller compared to the other cases. The significant differences between the behaviors of the temperature and velocity boundary layer shown in Fig. 10 is because the temperature drops as the flow accelerates and the $M a$ increases across the vane. However, the contribution of the $H_{M}$ term is much smaller compared to the major terms such as $P_{R}$ and $V_{M}$.

In order to quantify the contribution of the strong shock in the $M a=1.1$ case, we apply the shock-capturing method from Bogey et al. [19] to extract the region near the shock. The defi- 


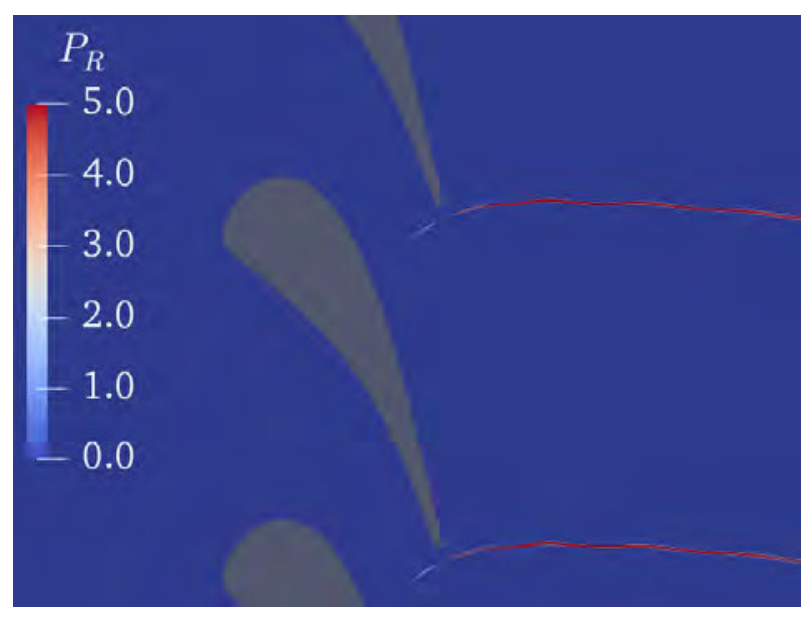

FIGURE 12. THE TURBULENCE PRODUCTION $P_{R}$ MASKED BY THE SHOCK CAPTURING $\sigma_{j}^{s c}$ IN THE $M a=1.1$ CASE.

nition of the shock-capturing coefficient $\sigma_{i}^{S C}$ is

$$
\begin{aligned}
D p_{j} & =\left(-P_{j+1}+2 P_{j}-P_{j-1}\right) / 4, \\
D p_{j}^{\text {magn }} & =\frac{1}{2}\left[\left(D p_{j}-D p_{j+1}\right)^{2}+\left(D p_{j}-D p_{j-1}\right)^{2}\right], \\
r_{j} & =\frac{D p_{j}^{\text {magn }}}{P_{j}^{2}}+\varepsilon, \\
\sigma_{j}^{s c} & =\frac{1}{2}\left(1-\frac{r_{t h}}{r_{j}}+\left|1-\frac{r_{t h}}{r_{j}}\right|\right) .
\end{aligned}
$$

Here, $P_{j}$ is the mean pressure on the $j^{\text {th }}$ grid point, and $\varepsilon=10^{-16}$ and $r_{t h}=10^{-6}$ are constants. It can therefore be deduced from Eqn. (2) that the shock region with strong mean pressure gradient has $\sigma_{j}^{s c}=1$ while the rest of the domain should have $\sigma_{j}^{s c}=0$. We remark here although a dilatation-based shock sensor, which is used in the present simulations, might be more suitable for distinguishing between shocks and turbulent fluctuations, the pressurebased sensor is used to quantify the shock-related entropy loss here. The reason is that applying the pressure-based method in Eqn. (2) is more straightforward, and the calculation of $\sigma_{i}^{S C}$ in this post-processing process is based on the averaged pressure field, in which the effects of turbulent fluctuations are negligible.

With this $\sigma_{j}^{s c}$ as a mask, we can extract the contribution of the shock region as shown in Fig. 12, and the integral of the entropy generation terms from the shock region is presented in Tab. 2. It is noted that the loss generated by the shock contributes around $6.8 \%$ of the total loss in the $M a=1.1$ case. Therefore, the major reason for the extra loss in the high Mach number case is the extensive turbulence production caused by the strong wake mixing near the blade trailing edge, rather than the shock itself.

\section{CONCLUSION}

We have performed a series of highly resolved large-eddy simulations of the LS89 HPT vane with exit Mach numbers varying from $M a=0.7$ to $M a=1.1$. To handle the complex flow physics caused by the strong shock in the $M a=1.1$ case, an overset mesh configuration was introduced, and the grid resolution was validated to be sufficiently fine to accurately resolve the boundary layer development. In addition, the synthetic turbulence inlet boundary condition was compared to 'realistic' turbulence generated by wakes of upstream cylindrical bars, showing that the artificial inlet turbulence is adequate and suitable for the prediction of HPT performance.

Based on the high-fidelity data from the present simulations, a detailed analysis of the flow fields shows that the varying exit Mach number can significantly change the flow physics and thus the turbine efficiency by affecting the suction-side transition, blade boundary layer state, and wake mixing. To further understand the physical mechanisms responsible for the Mach number effects on the kinetic loss, the entropy breakdown method [11] has been applied to the HPT data, and the different entropy generation terms have been quantitatively analyzed. It is shown that the higher-level loss in the high $M a$ case is mainly because of the strong wake mixing near the blade trailing edge and viscous dissipation in the blade boundary layer, whereas the additional loss caused by the shock itself is relatively small.

\section{ACKNOWLEDGMENT}

This research used resources of the Oak Ridge Leadership Computing Facility, which is a DOE Office of Science User Facility supported under Contract DE-AC05-00OR22725. This work was also supported by a grant from the Swiss National Supercomputing Centre (CSCS) under project ID s977.

\section{REFERENCES}

[1] Arts, T., Lambertderouvroit, M., and Rutherford, A. W., 1990. Aero-thermal Investigation of a Highly Loaded Transonic Linear Turbine Guide Vane Cascade. Tech. rep., von Karman Institute for Fluids Dynamics, Brussels, Belgium.

[2] Sieverding, C. H., Arts, T., Dénos, R., and Martelli, F., 1996. "Investigation of the Flow Field Downstream of a Turbine Trailing Edge Cooled Nozzle Guide Vane". ASME J. Turbomach., 118(2), pp. 291-300.

[3] Pichler, R., Sandberg, R. D., Michelassi, V., and Bhaskaran, R., 2016. "Investigation of the Accuracy of RANS Models to Predict the Flow Through a Low-Pressure Turbine". ASME J. Turbomach., 138(12), p. 121009.

[4] Sandberg, R. D., and Michelassi, V., 2019. "The Current State of High-Fidelity Simulations for Main Gas Path Turbomachinery Components and Their Industrial Impact". Flow Turbul. Combust., 102(4), pp. 797-848. 
[5] Pichler, R., Sandberg, R. D., Laskowski, G., and Michelassi, V., 2017. "High-Fidelity Simulations of a Linear HPT Vane Cascade Subject to Varying Inlet Turbulence". In ASME Turbo Expo 2017: Turbomachinery Technical Conference and Exposition, Paper No. GT201763079.

[6] Zhao, Y., and Sandberg, R. D., 2019. "Bypass Transition in Boundary Layers Subject to Strong Pressure Gradient and Curvature Effects". J. Fluid Mech.(in revision).

[7] Bhaskaran, R., and Lele, S. K., 2010. "Large Eddy Simulation of Freestream Turbulence effects on Heat Transfer to a High-Pressure Turbine Cascade”. J. Turbul., 11(6), pp. $1-15$.

[8] Gourdain, N., Gicquel, L., and Collado, E., 2012. "Comparison of RANS and LES for Prediction of Wall Heat Transfer in a Highly Loaded Turbine Guide Vane". J. Propuls. Power, 28(2), pp. 423-433.

[9] Segui, L., Gicquel, L., Duchaine, F., and de Laborderie, J., 2017. "LES of the LS89 Cascade: Influence of Inflow Turbulence on the Flow Predictions". In Proceedings of 12th European Conference on Turbomachinery Fluid dynamics and Thermodynamics ETC12, pp. 3-7.

[10] Harnieh, M., Gicquel, L., and Duchaine, F., 2017. "Sensitivity of Large Eddy Simulations to Inflow Condition and Modeling If Applied to a Transonic High-Pressure Cascade Vane". In ASME Turbo Expo 2017: Turbomachinery Technical Conference and Exposition, Paper No. GT201764686.

[11] Zhao, Y., and Sandberg, R. D., 2019. “Using a New Entropy Loss Analysis to Assess the Accuracy of RANS Predictions of an HPT Vane". In ASME Turbo Expo 2019: Turbomachinery Technical Conference and Exposition, Paper No. GT2019-90126.

[12] Sandberg, R. D., Michelassi, V., Pichler, R., Chen, L., and Johnstone, R., 2015. "Compressible Direct Numerical Simulation of Low-Pressure Turbines-Part I: Methodology". ASME J. Turbomach., 137(5), p. 051011.

[13] Kim, J. W., and Sandberg, R. D., 2012. "Efficient Parallel Computing with a Compact Finite Difference Scheme". Comput. Fluids, 58, pp. 70-87.

[14] Kennedy, C. A., Carpenter, M. H., and Lewis, R. M., 2000. "Low-Storage, Explicit Runge-Kutta Schemes for the Compressible Navier-Stokes Equations". Appl. Numer. Math., 35(3), pp. 177-219.

[15] Michelassi, V., Chen, L., Pichler, R., and Sandberg, R. D., 2015. "Compressible Direct Numerical Simulation of LowPressure TurbinesPart II: Effect of Inflow Disturbances". ASME J. Turbomach., 137(7), p. 071005.

[16] Wheeler, A. P. S., Sandberg, R. D.and Sandham, N. D., Pichler, R., and Michelassi, V., 2016. "Direct Numerical Simulations of a High-Pressure Turbine Vane". ASME J. Turbomach., 138(7), p. 071003.
[17] Pichler, R., Zhao, Y., Sandberg, R. D., Michelassi, V., Pacciani, R., Marconcini, M., and Arnone, A., 2018. "LES and RANS Analysis of the End-Wall Flow in a Linear LPT Cascade: Part IFlow and Secondary Vorticity Fields Under Varying Inlet Condition". In ASME Turbo Expo 2018: Turbomachinery Technical Conference and Exposition, Paper No. GT2018-76233.

[18] Marconcini, M., Pacciani, R., Arnone, A., Michelassi, V., Pichler, R., Zhao, Y., and Sandberg, R. D., 2019. "'Large Eddy Simulation and RANS Analysis of the End-Wall Flow in a Linear Low-Pressure-Turbine Cascade - Part II: Loss Generation". ASME J. Turbomach., 141(5), p. 051004.

[19] Bogey, C., De Cacqueray, N., and Bailly, C., 2009. "A Shock-Capturing Methodology Based on Adaptative Spatial Filtering for High-Order Non-Linear Computations". $J$. Comp. Phys., 228(5), pp. 1447-1465.

[20] Kim, J. W., and Lee, D. J., 2003. "Characteristic Interface Conditions for Multiblock High-Order Computation on Singular Structured Grid". AIAA J., 41(12), pp. 23412348.

[21] Chesshire, G., and H., W. D., 1990. "Composite Overlapping Meshes for the Solution of Partial Differential Equations". J. Comput. Phys., 90(1), pp. 1-64.

[22] Klein, M., Sadiki, A., and Janicka, J., 2003. "A Digital Filter Based Generation of Inflow Data for Spatially Developing Direct Numerical or Large Eddy Simulations". $J$. Comput. Phys., 186(2), pp. 652-665.

[23] Nicoud, F., and Ducros, F., 1999. "Subgrid-Scale Stress Modelling Based on the Square of the Velocity Gradient Tensor". Flow Turbul. Combust., 62(3), pp. 183-200.

[24] Sandberg, R. D., and Sandham, N. D., 2006. "Nonreflecting Zonal Characteristic Boundary Condition for Direct Numerical Simulation of Aerodynamic Sound". AIAA J., 44(2), pp. 402-405.

[25] Wu, X., 2017. "Inflow Turbulence Generation Methods". Annu. Rev. Fluid Mech., 49, pp. 23-49.

[26] Denton, J. D., 1993. "Loss Mechanisms in Turbomachines". ASME J. Turbomach., 115(4), p. 621. 


\section{University Library}

\section{$\bullet-\underset{A C C E S S}{M} \operatorname{N} E R V A$}

\section{A gateway to Melbourne's research publications}

Minerva Access is the Institutional Repository of The University of Melbourne

Author/s:

Zhao, Y;Sandberg, R

Title:

HIGH-FIDELITY SIMULATIONS OF A HIGH-PRESSURE TURBINE VANE SUBJECT TO LARGE DISTURBANCES: EFFECT OF EXIT MACH NUMBER ON LOSSES

Date:

2021

Citation:

Zhao, Y. \& Sandberg, R. (2021). HIGH-FIDELITY SIMULATIONS OF A HIGH-PRESSURE TURBINE VANE SUBJECT TO LARGE DISTURBANCES: EFFECT OF EXIT MACH NUMBER ON LOSSES. Proceedings of ASME Turbo Expo 2020 Turbomachinery Technical Conference and Exposition GT202, 2B-2020, Search Results Web results ASME: The American Society of Mechanical Engineers. https://doi.org/10.1115/GT2020-14445.

Persistent Link:

http://hdl.handle.net/11343/241912 